\title{
Localization system in GPS-denied environments using radar and IMU measurements: application to a smart white cane
}

\author{
Jeremy Barra ${ }^{1,3}$, Suzanne Lesecq ${ }^{1}$, Mykhailo Zarudniev ${ }^{1}$, Olivier Debicki ${ }^{2}$, Nicolas Mareau ${ }^{1}$, Laurent Ouvry ${ }^{1}$
}

\begin{abstract}
This paper presents the development of a localization system in GPS-denied environments using an Inertial Measurement Unit (IMU) and a Pulse-Doppler radar. A ground speed estimation from radar measurements is first proposed. This estimation is combined with noisy measurements from an IMU in a Luenberger observer, allowing accurate deadreckoning. The methodology proposed provides short-term position of the sensors embedded in a white cane, the ultimate goal being obstacle detection through the computation of a model of the surroundings. The results show that this solution gives an error growth rate of the position estimation of $0.026 \mathrm{~m} / \mathrm{s}$, which is a hundred times better than the one obtained with the naive double integration of the accelerometer data.
\end{abstract}

\section{INTRODUCTION}

According to the World Health Organization, 1.3 billion people are estimated to be visually impaired worldwide. With regards to distance vision, about 405 million have low vision and 36 million are blind [11]. For these people, the risks of falls and collisions in unknown environments is increased. About $40 \%$ of them suffer from a head level accident and $30 \%$ suffer a fall accident at least once a month [9]. In this context, Electronic Travel Aids (ETA) could help to improve these statistics by providing to the visually impaired information about their environment.

ETA examples such as smart white canes embedding an environment perception system can be found in the literature [5]. These solutions provide more information to the users about their environment than a standard white cane. In such systems, obstacle detection is often performed using only distance measurements from sensors such as ultrasound or lidar. Other solutions use a radar to detect and localize stationary and moving obstacles [3] [12].

In the context of the INSPEX project [13], several sensors including a Pulse-Doppler radar and an IMU are embedded on a white cane. Note that the IMU contains 3-axis accelerometer, gyroscope and magnetometer. The measurements of the sensors embedded on the cane are used to produce a model of the user's surroundings using occupancy grids [14]. An accurate computation of the occupancy grid requires the knowledge of the sensors position and their orientation over the observation time.

The present paper proposes an estimation method of the position of the embedded sensors that do not rely on strong

\footnotetext{
${ }^{1}$ Univ. Grenoble Alpes, CEA, LETI, DACLE, LIALP, F-38000 Grenoble, France, ${ }^{2}$ Univ. Grenoble Alpes, CEA, LIST, DACLE, LIALP, F-38000 Grenoble, France, ${ }^{3}$ Laboratoire Ampère, Université de Lyon, École Centrale de Lyon, 69134 Écully Cedex, France
}

hypotheses regarding the white cane motion. To this end, we propose to combine the noisy and biased measurements from the IMU with information provided by the Pulse-Doppler radar. Usually, radar measurements are used to estimate the relative speed between the radar and the obstacles thanks to the Doppler effect. Here we propose to go a step ahead and to use these measurements to determine the radar ground speed. This estimation is then combined with IMU measurements to estimate the position of the sensors embedded on the white cane in 3 dimensions (3D).

In the next section, a short overview of the previous work on the localization in GPS-denied environments is given. Section III presents the referential frames and sensors used in this study. Then, a method to estimate the speed of the radar with respect to the ground is introduced in section IV. This speed estimate is merged with information extracted from the IMU to improve the estimation of the sensors position in 3D. The whole approach being implemented on a microcontroller embedded in the white cane, a Luenberger observer has been designed to merge the information in a computing-efficient way in section V. Finally, section VI presents the experimental results of this position estimation method which highly improves the position estimate.

\section{RELATED WORK}

Several solutions for localization in GPS-denied environments have been presented in the literature, especially for indoor pedestrian navigation [6]. To ensure the versatility of the proposed solution, focus is given here to infrastructure-free localization techniques also called deadreckoning techniques. These solutions are mainly based on IMU measurements. They can be divided into two groups: Inertial Navigation Systems (INS) and Step-and-HeadingSystem (SHS).

The INS method consists in computing the position of the IMU from the double integration of the acceleration measured in 3D. Due to the sensor noise and bias drift, the error on the position estimation grows quickly, especially when low-cost IMU are used. A common approach for pedestrian navigation is a foot-mounted IMU configuration, which constrains the movement of the sensor. Basically, the foot can be considered periodically stationary while the user is walking. This information is combined with the current position and velocity estimate to reset the velocity hence correct the state estimate. This technique called zero-velocity update (ZUPT) [10] helps to reduce the error growth. A 
similar approach adapted for the white cane can be found in [1]. In this paper, the white cane motion (called swing) is described. It is shown that a stationary interval can be found between each swing of the cane, when the gyro measurements for the three axes are approximately zero. A temporary coordinate frame for each swing is defined from the accelerometer measurements under the assumption that during a walk at constant speed, the IMU measures only gravity at stationary points.

The SHS method is specific to pedestrians and gives a position estimate only in two dimensions. After detecting the stationary interval of a step, i.e. when the gyro measurements norm is approximately zero, the new position of the pedestrian is computed given the step length and its heading. This approach can be adapted to the white cane by observing that at each step, the user of a white cane usually performs one swing which can be detected as explained previously from gyro measurements.

The white cane motion is unconstrained and there are different situations in which the user can be found other than stable walk. The solutions proposed in the literature often rely on strong assumptions on the motion of the mobile, e.g. on the step length of the user or the swing motion of the white cane. However, these assumptions can easily be unsatisfied. For example, the step of a user walking in a crowded environment will be shorter than in normal situation. For this reason a hypothesis-based dead-reckoning approach such as a white cane adapted SHS or ZUPT-INS algorithm is not suitable in the present study.

\section{PROBLEM CONFIGURATION}

\section{A. Frames of reference}

Three frames are used in this study, they are represented on figure 1. Under the hypothesis of a flat ground surface, they are defined as:

- $\mathcal{R}_{0}=\left(O, \overrightarrow{x_{0}}, \overrightarrow{y_{0}}, \overrightarrow{z_{0}}\right)$ is a local reference frame in which the sensors location has to be determined. Its plan $\left(\overrightarrow{x_{0}} O \overrightarrow{y_{0}}\right)$ is tangential with the ground.

- $\mathcal{R}=\left(O^{\prime}, \vec{x}, \vec{y}, \vec{z}=\overrightarrow{z_{0}}\right)$ is a referential attached to the user of the white cane. Its origin is a projection of the center of gravity of the user on the ground. Its $\vec{y}$ axis is oriented in the direction of the user's walk such that there is a rotation between $\mathcal{R}_{0}$ and $\mathcal{R}$ of angle $\psi$ around the $\vec{z}$ axis.

- $\mathcal{R}_{b}=\left(O_{b}, \overrightarrow{x_{b}}, \overrightarrow{y_{b}}, \overrightarrow{z_{b}}\right)$ is the referential attached to the body of the sensors.

\section{B. Inertial Measurement}

An IMU usually consists in 3-axes sensors of 3 modalities, namely, accelerometer, gyroscope and magnetometer that measure respectively the sensor acceleration, the angular velocity and the local magnetic field in the sensor body referential $\mathcal{R}_{b}$. It has been shown in the literature that the measurements from these three sensors can be merged to estimate the absolute orientation measurement of the IMU [4]. In the present work, the estimation of the IMU orientation is not addressed as the IMU mounted on the cane

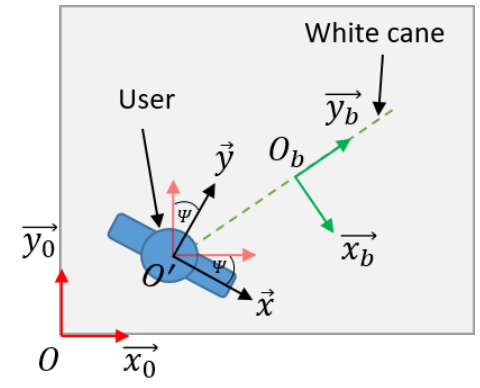

Fig. 1: Frames of reference

already uses a state of the art attitude estimation algorithm. Therefore, the measurements in $\mathcal{R}_{b}$ can be expressed in $\mathcal{R}_{0}$ using a rotation matrix $R_{0}^{b}$ defined from the estimated attitude. For the position estimation of the sensors, the linear acceleration is expressed in the $\mathcal{R}_{0}$ frame. This corresponds to the acceleration measured by the accelerometers without the influence of gravity.

\section{Pulse-Doppler radar}

The beam emitted by the radar can be assimilated to a cone named "field of view" (FOV). The radar is mounted on the white cane such that the axis of that FOV intersects the ground with angle $\alpha_{0}$ and coincides with the $\overrightarrow{y_{b}}$ axis of the IMU. With this configuration, the angle $\alpha_{0}$ is estimated from the IMU measurements as the pitch angle. Figure 2 represents the IMU mounted on the white cane as well as the FOV of the radar as a right circular cone of an aperture angle $\delta$ which depends of the used antenna.

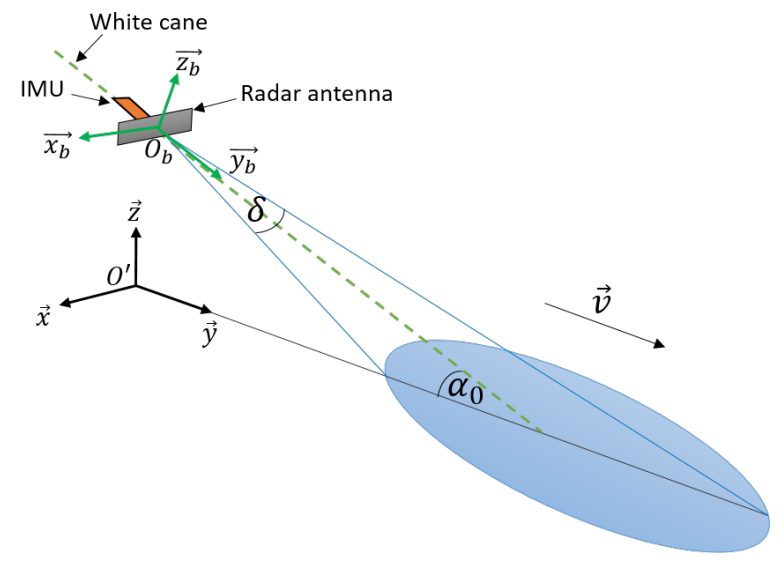

Fig. 2: White cane sensors configuration

\section{Data fusion procedure overview}

The measurements from these two sensors are used to obtain an estimation of their position, i.e. the position of $O_{b}$ in the $\mathcal{R}_{0}$ frame. First, the attitude computed from the IMU measurements and the raw signal from the radar are processed through a ground speed estimation algorithm to estimate the velocity of the sensors in $\mathcal{R}_{0}$. Then, this estimation is combined with the linear acceleration estimated from the IMU measurements and expressed in $\mathcal{R}_{0}$. This 
provides an estimation of the position of the sensors in frame $\mathcal{R}_{0}$. The whole procedure is illustrated in figure 3 .

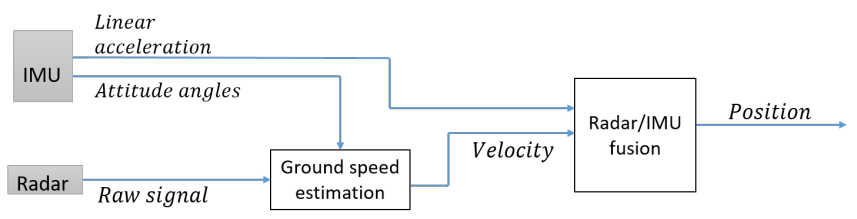

Fig. 3: Illustration of the sensors position estimation algorithm

\section{GROUND-SPEED ESTIMATION}

The following section presents the proposed method to estimate the ground-speed $v$ of the sensors mounted on the white cane using raw Pulse-Doppler measurements. The different steps of this method are summarized on figure 4 .

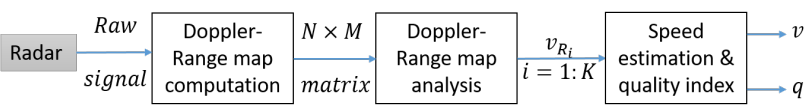

Fig. 4: Ground-speed estimation steps

\section{A. From raw measurements to Range-Doppler map}

The Pulse-Doppler radar emits coherent pulses at the pulse repetition frequency through its antenna. Each time a pulse is sent by the radar, its energy is reflected by the environment, here, the ground. The reflection is returned to the antenna with a time delay:

$$
\tau=2 \frac{R}{c} \Leftrightarrow R=\frac{c \tau}{2}
$$

$R$ is the distance from the antenna to the ground and $c=$ $3.10^{8} \mathrm{~m} . \mathrm{s}^{-1}$ is the electromagnetic wave speed in vacuum. According to (1), the time axis in the received time-domain signal can be replaced by a range axis. This allows to express the energy received as a function of the distance from the radar to the obstacle (the ground), which is sampled in $M$ values, see figure 5 .

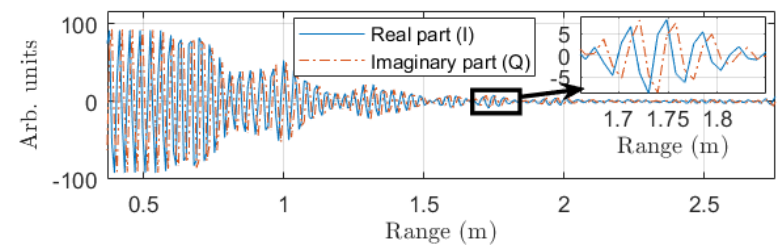

Fig. 5: Single time-domain measurement

The acquisition of $N$ time-domain measurements gives a $N \times M$ time-domain matrix. A Fast Fourier Transform is then applied to this matrix, leading to a frequency-domain spectrum, sometimes called Range-Doppler map [8] shown at figure 6.

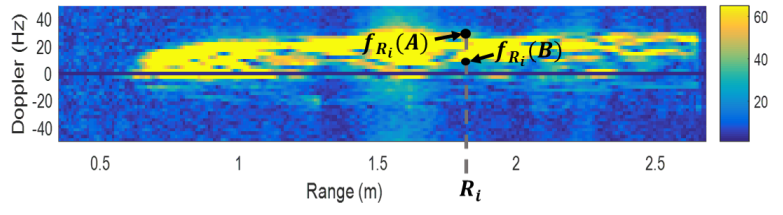

Fig. 6: Range-Doppler map representation

\section{B. Range-Doppler map analysis}

Figure 6 shows that the frequency-domain spectrum is spread in the range and Doppler direction. The spreading in the range direction is caused by the aperture $\delta$ of the FOV in the $\left(\vec{y} O^{\prime} \vec{z}\right)$ plan, see figure 7 . In this plan, the FOV is discretized in $K$ rays intersecting the ground with an angle $\alpha_{R_{i}} \in\left[\alpha_{0}-\frac{\delta}{2} ; \alpha_{0}+\frac{\delta}{2}\right], i=1: K$.

Side

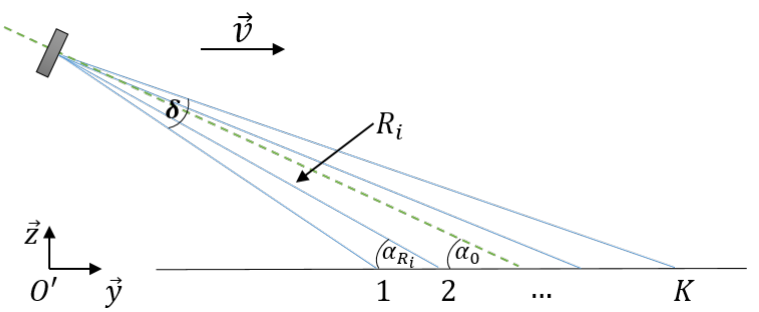

Fig. 7: Discretization of the field-of-view (side view)

In this configuration, the ground speed $v_{R_{i}}$ perceived along a ray $R_{i}$ is given by the formula:

$$
v_{R_{i}}=\frac{c f_{R_{i}}}{2 f_{t r} \cos \left(\alpha_{R_{i}}\right)}, i=1: K
$$

where $f_{t r}$ is the transmitted frequency, $f_{R_{i}}$ is the Doppler frequency received at a given range $R_{i}$ and $\alpha_{R_{i}}$ is the angle between the ray $R_{i}$ and the ground, see figure 7 .

The spreading of the frequency-domain spectrum in the Doppler direction is due to the aperture angle $\delta$ in the plan of the ground $\left(\vec{x} O^{\prime} \vec{y}\right)$. At a given point at range $R_{i}$ (say $\mathrm{B}$ on figure 8), a projection of the velocity is measured instead of its real value measured at point $A$.

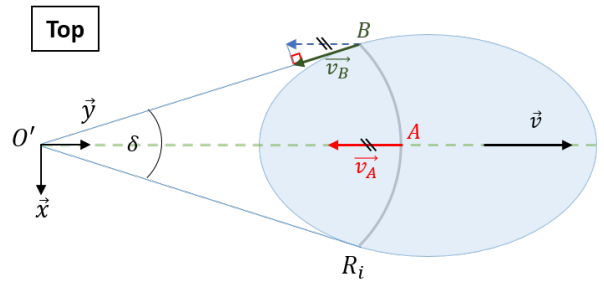

Fig. 8: Top view of the field-of-view

As a result, the Doppler frequency perceived for a given range $R_{i}$ is not unique. The frequency corresponding to the non-projected velocity will be found on the superior edge of the frequency-domain spectrum, see Doppler frequencies associated to points $A$ and $B$ on figure 6. The Doppler 
frequency $f_{R_{i}}$ at a range $R_{i}$ used in (2) is then determined with a standard edge-detection technique based for instance on the determination of the maximum gradient [2].

Note that the solution proposed supposes that the velocity vector $\vec{v}$ and the line of sight of the radar are both included in the $\left(\vec{y} O^{\prime} \vec{z}\right)$ plan. This is not always the case as the white cane is subject to a swing movement [1] induced by the user to scan the ground in front of him. However, the superior edge of the frequency-domain spectrum can still provide the nonprojected velocity value. This is verified under the condition that the angle $\theta$ between the velocity $\vec{v}$ direction and the line of sight does not exceed $\frac{\delta}{2}$, as shown on figure 9 .

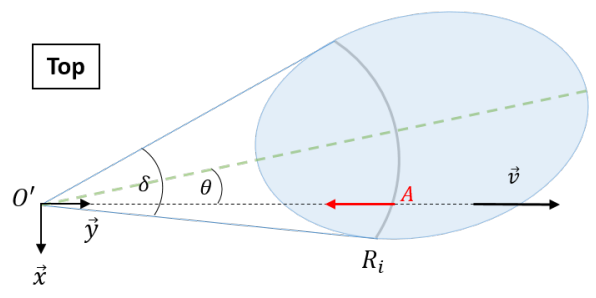

Fig. 9: Non-projected velocity value still in the FOV

\section{Speed estimation and quality index}

From the $K$ rays chosen at different ranges in the FOV, $K$ independent speed estimations are obtained from (2) after detecting the superior edge of the frequency-domain spectrum at these given ranges. The redundancy of information that comes from the aperture $\delta$ allows to filter eventual perturbations in the FOV. The final speed estimation is obtained as a function of the $K$ values of $v_{R_{i}}$. In this work, $f$ is the mean of the $K$ values of $v_{R_{i}}$.

$$
v=f\left(v_{R_{i}}\right), i=1: K
$$

A quality index can also be given as a function $g$ of $v_{R_{i}}$. For instance, $g$ can correspond to the standard deviation of the $K$ values of $v_{R_{i}}$.

$$
q=g\left(v_{R_{i}}\right), i=1: K
$$

\section{RADAR \& IMU INFORMATION COUPLING}

Coupling the ground-speed estimate proposed above with the information extracted from IMU measurements allows to address a large variety of movements of the white cane. This section shows how information extracted from both modalities can be merged to efficiently estimate the sensors position in $3 \mathrm{D}$.

\section{A. Geometrical configuration}

The ground speed determined with the Doppler radar is oriented using the yaw angle estimated by the IMU. This is done under the assumption that the line of sight of the cane and the velocity $\vec{v}$ are included in the same plan $\left(\vec{y} O^{\prime} \vec{z}\right)$ as represented on figure 8 . Note with this assumption, there is an uncertainty on the heading angle of the velocity of $\pm \frac{\delta}{2}$.
We finally obtain an estimation of the velocity of the white cane $\vec{v}$ from the speed over ground $v$ estimated as:

$$
\vec{v}=\left[\begin{array}{c}
v \cos \psi \\
v \sin \psi \\
0
\end{array}\right]
$$

where $\psi$ is the yaw angle computed from by IMU measurements.

\section{B. Linear discrete-time state-space model}

To address a large variety of possible movements of the white cane, a general model of the sensors motion derived from the standard dynamics equations is chosen. The statespace model used to estimate the sensors position is given by:

$$
\begin{aligned}
{\left[\begin{array}{l}
a_{k} \\
v_{k} \\
p_{k}
\end{array}\right] } & =\left[\begin{array}{ccc}
I_{3} & 0_{3} & 0_{3} \\
T_{s} \cdot I_{3} & I_{3} & 0_{3} \\
0_{3} & T_{s} \cdot I_{3} & I_{3}
\end{array}\right]\left[\begin{array}{l}
a_{k-1} \\
v_{k-1} \\
p_{k-1}
\end{array}\right] \\
y_{k} & =\left[\begin{array}{lll}
I_{3} & 0_{3} & 0_{3} \\
0_{3} & I_{3} & 0_{3}
\end{array}\right]\left[\begin{array}{l}
a_{k} \\
v_{k} \\
p_{k}
\end{array}\right]
\end{aligned}
$$

where $T_{s}$ is the sampling period, $I_{3}$ represents the $3 \times 3$ identity matrix. $a_{k} \in \mathbb{R}^{3}$ is the linear acceleration of the sensors mounted on the white cane in the $\mathcal{R}_{0}$ frame, which corresponds to the acceleration deprived from its gravity component. $v_{k} \in \mathbb{R}^{3}$ and $p_{k} \in \mathbb{R}^{3}$ are respectively the velocity and the position in the $\mathcal{R}_{0}$ frame. In this model, the linear acceleration of the white cane is considered constant over the sampling period. This assumption is valid because the IMU measurements are acquired at frequency of $100 \mathrm{~Hz}$ which is much higher than the motion dynamics.

Note however that with this model, the full-state is not observable. Therefore, we use the reduced state $x_{k}$ :

$$
x_{k}=\left[\begin{array}{l}
a_{k} \\
v_{k}
\end{array}\right]
$$

The position will consequently only be predicted by integrating the state $x_{k}$. The system becomes:

$$
\begin{aligned}
{\left[\begin{array}{l}
a_{k} \\
v_{k}
\end{array}\right] } & =\overbrace{\left[\begin{array}{cc}
I_{3} & 0_{3} \\
T_{s} \cdot I_{3} & I_{3}
\end{array}\right]}^{A}\left[\begin{array}{l}
a_{k-1} \\
v_{k-1}
\end{array}\right] \\
y_{k} & =\underbrace{\left[\begin{array}{ll}
I_{3} & 0_{3} \\
0_{3} & I_{3}
\end{array}\right]}_{C} x_{k}
\end{aligned}
$$

The state $x_{k}$ is now fully measured, $a_{k}$ being obtained from the IMU while $v_{k}$ comes from the radar data processing.

\section{Multisensor data fusion}

In the present work, the measurements $a_{k}$ and $v_{k}$ are combined using a Luenberger observer [7]:

$$
\left\{\begin{aligned}
\hat{x}_{k+1} & =A \hat{x}_{k}+L\left[y_{k}-\hat{y}_{k}\right] \\
\hat{y}_{k} & =C \hat{x}_{k}
\end{aligned}\right.
$$


where $\hat{x}$ is the estimated reduced state and $y_{k}$ is the vector of sensors "pseudo-measurements" at time $k$. $L$ is the observer gain, determined by pole-placement such that the matrix $A-$ $L C$ has all its eigenvalues inside the unit circle.

In the literature, the localization problem using on-board sensors such as an IMU is usually solved with a Kalman filter, considering the equation 8 in a nondeterministic case:

$$
\begin{aligned}
{\left[\begin{array}{l}
a_{k} \\
v_{k}
\end{array}\right] } & =\left[\begin{array}{cc}
I_{3} & 0_{3} \\
T_{s} \cdot I_{3} & I_{3}
\end{array}\right]\left[\begin{array}{l}
a_{k-1} \\
v_{k-1}
\end{array}\right]+\left[\begin{array}{l}
{ }^{a} \vec{w}_{k} \\
{ }^{v} \vec{w}_{k}
\end{array}\right] \\
y_{k} & =\left[\begin{array}{ll}
I_{3} & 0_{3} \\
0_{3} & I_{3}
\end{array}\right] x_{k}+\left[\begin{array}{l}
{ }^{a} \vec{v}_{k} \\
v \\
v_{k}
\end{array}\right]
\end{aligned}
$$

where ${ }^{a} \vec{w}_{k},{ }^{v} \vec{w}_{k}$ and ${ }^{a} \vec{v}_{k},{ }^{v} \vec{v}_{k}$ are respectively the process and the measurement Gaussian white noises.

Such a solution has been also implemented and the results obtained were similar, see table I. The main drawback of the Kalman filter solution, is that it requires more computational workload (e.g. computation of a gain at each sampling time). As the proposed solution will be integrated on a low computing capability microcontroller with fixed-point arithmetic, the Luenberger observer seems to be a better option from a computational point-of-view.

\section{RESULTS}

\section{A. Experimental setup}

The experiments were carried out with a prototype of the white cane. An IMU Bosch BNO055 and a Timedomain Doppler radar Ultra-Wide band P440 were mounted on the cane (see figure 10), both being sampled at $100 \mathrm{~Hz}$.

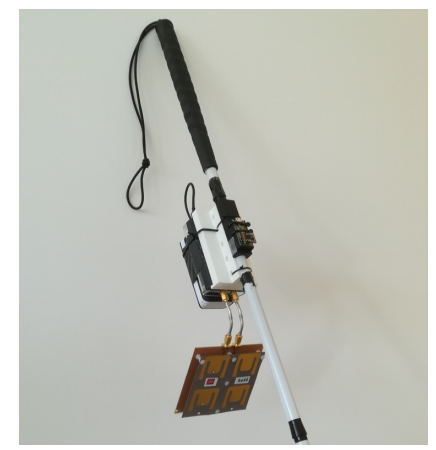

Fig. 10: Prototype of the electronic white cane

The experiments were conducted indoor. A walk was performed on a closed rectangular-shaped $8 m \times 3 m$ loop, with a length of $22 \mathrm{~m}$. In the first set of experiments presented hereafter, data was acquired on a computer and post-treated on Matlab.

During the test, we didn't have access to an indoor position tracking equipment. Therefore, the criterion used to measure the performance of the proposed solution is the final distance error to the starting point.

\section{B. Comparison of estimation algorithms}

The radar/IMU coupling solutions based on a Kalman filter and a Luenberger observer are compared with two state of the art approaches, namely, a naive double integration of the linear acceleration (naive INS), a Step-Heading-System algorithm adapted for the white cane (adapted SHS).

The adapted SHS approach relies on strong hypotheses on the movement. The main one is to consider that a step of the user corresponds to a swing of the white cane. At the end of a swing, the angular velocity of the sensors becomes null as the next swing will be performed in the opposite direction. This instant, called stationary point, is detected thanks to the gyroscope measurement norm that is approximately zero. The velocity of the sensors $\vec{v}$ at this point is then the velocity

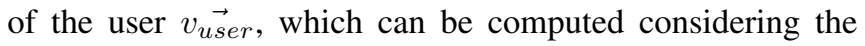
average step length of the user and the elapsed time between stationary points. Figure 11 represents the sensors trajectory during the swing.

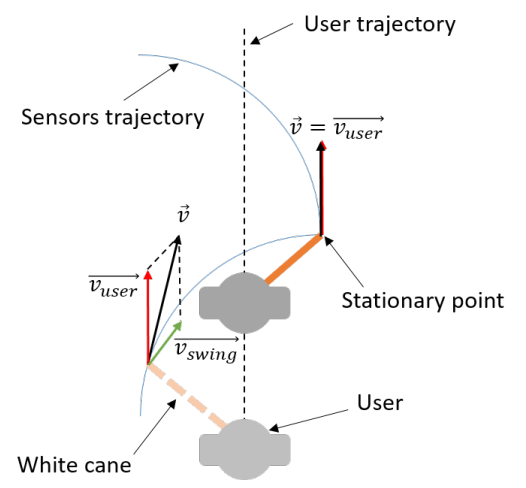

Fig. 11: Motion hypothesis for the adapted SHS algorithm

\section{Experimental results}

The results from a first experiment where the swing movement is performed at each step of the user are presented on figure 12. It shows that the position estimation from the naive double integration of the linear acceleration quickly drifts as expected. As the hypotheses on the movement for the SHS algorithm are fulfilled, the trajectory obtained with this approach is similar to the ones obtained with the coupling of the radar and the IMU. In this case, the adapted SHS solution gives the smaller final distance error, $0.9 \mathrm{~m}$, whereas the radar/IMU algorithms based on a Luenberger observer and on a Kalman filter give respectively a $1.4 \mathrm{~m}$ and $1.5 m$ final distance error.

Another experiment is presented on figure 13. Note that the swing of the cane is stopped at some point. The hypotheses on the movement for the adapted SHS are not fulfilled anymore at this time, leading to a poor quality of the position estimate. However, the radar/IMU Luenberger and Kalman approaches still give final distance errors similar to the ones of the previous experiment, respectively $1 \mathrm{~m}$ and $1.3 \mathrm{~m}$. This experiment shows the versatility of the radar/IMU coupling solutions, which do not rely on strong assumptions on the white cane motion.

Ten experiments have been carried out on the path of length $22 \mathrm{~m}$ with an average travel time of 50 seconds. The white cane swing is performed at each step, to fulfil the 


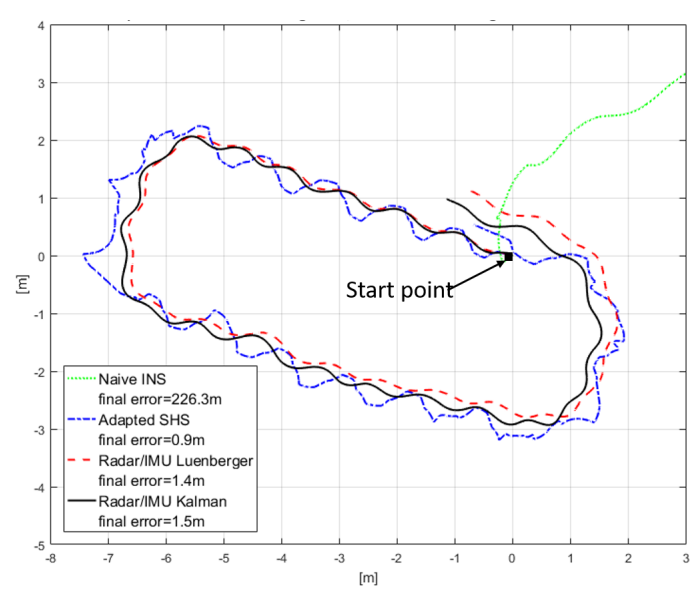

Fig. 12: Swing movement on the whole path

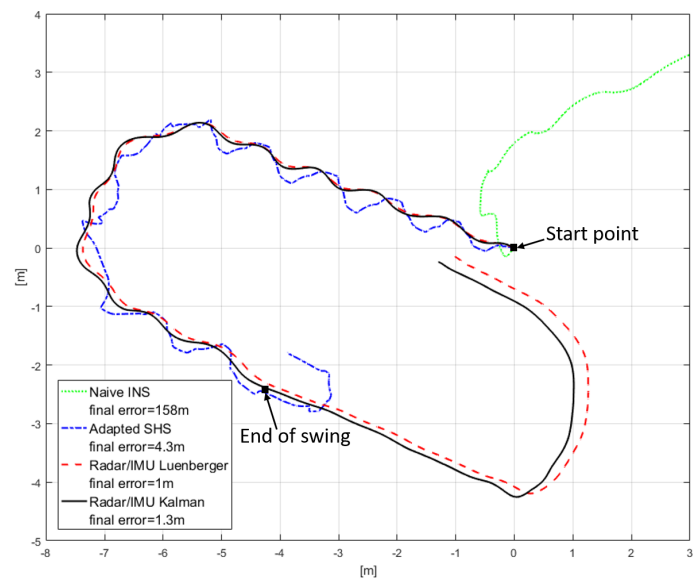

Fig. 13: Swing movement stopped midway

hypotheses of the adapted SHS approach. The final distance errors are summarised on table I. Even though the hypotheses of the adapted SHS algorithm are fulfilled, the radar/IMU coupling algorithms give better results and reduce the final distance error. We note that the solution based on a Kalman filter gives close results to the one based on a Luenberger observer. The latter is the solution chosen to be implemented in the future on the microcontroller, as explained in section V-C. Finally, the error growth rate for the chosen solution is $0.026 \mathrm{~m} / \mathrm{s}$ which is a hundred times better than the one of the naive double integration approach.

\begin{tabular}{|l|l|l|l|l|}
\hline & $\begin{array}{l}\text { Radar/IMU } \\
\text { Luenberger }\end{array}$ & $\begin{array}{l}\text { Radar/IMU } \\
\text { Kalman }\end{array}$ & $\begin{array}{l}\text { Adapted } \\
\text { SHS }\end{array}$ & Naive INS \\
\hline Error max & $2.7 m$ & $2.7 m$ & $3.9 m$ & $246 m$ \\
\hline Error sd $\sigma$ & $0.5 m$ & $0.4 m$ & $0.9 m$ & $74 m$ \\
\hline Error mean & $1.3 m$ & $1.3 m$ & $1.9 m$ & $132 m$ \\
\hline $\begin{array}{l}\text { Error growth } \\
\text { rate }\end{array}$ & $0.026 m / s$ & $0.026 m / s$ & $0.038 m / s$ & $2.64 m / s$ \\
\hline
\end{tabular}

TABLE I: Final distance error on 10 experiments

\section{CONCLUSION}

This paper proposes a localization technique for GPSdenied environments. It aims to obtain a position estimation of the sensors mounted on a white cane. This method merges noisy IMU measurements with a velocity estimation obtained from Pulse-Doppler radar measurements. This latter is produced using a ground speed estimation algorithm which can also give a quality index of the speed estimation. The results show that the final distance mean error on a path of a length of $22 \mathrm{~m}$ of the radar/IMU solution is $1.3 \mathrm{~m}$. The error growth rate of this solution is $0.026 \mathrm{~m} / \mathrm{s}$, which is a hundred times smaller than the one of a naive double integration of the accelerometer data approach. The position estimate will be helpful in the future to build a model of the user's surroundings from the measurements of the sensors mounted on the white cane. This model will finally be used to enhance the travel capabilities of the users by guiding them in between the obstacles using an audio feedback.

\section{ACKNOWLEDGEMENT}

The work presented in this paper was carried out in the context of the H2020 project INSPEX funded by the European Union's Horizon 2020 research and innovation program under grant agreement No.730953. It was also supported by the French national program "Programme d'Investissements d'Avenir, IRT Nanoelec", ANR-10-AIRT-05.

\section{REFERENCES}

[1] Quoc Khanh Dang, Youngjoon Chee, Duy Duong Pham, and Young Soo Suh. A virtual blind cane using a line laser-based vision system and an inertial measurement unit. Sensors, 16(1):95, 2016.

[2] Larry S Davis. A survey of edge detection techniques. Computer graphics and image processing, 4(3):248-270, 1975.

[3] Valentina Di Mattia, Giovanni Manfredi, Alfredo De Leo, Paola Russo, Lorenzo Scalise, Graziano Cerri, Alina Caddemi, and Emanuele Cardillo. A feasibility study of a compact radar system for autonomous walking of blind people. In Research and Technologies for Society and Industry Leveraging a better tomorrow (RTSI), 2016 IEEE 2nd International Forum on, pages 1-5. IEEE, 2016.

[4] Estefania Munoz Diaz, Fabian de Ponte Müller, Antonio R Jiménez, and Francisco Zampella. Evaluation of ahrs algorithms for inertial personal localization in industrial environments. In Industrial Technology (ICIT), 2015 IEEE International Conference on, pages 3412-3417. IEEE, 2015.

[5] Wafa Elmannai and Khaled Elleithy. Sensor-based assistive devices for visually-impaired people: current status, challenges, and future directions. Sensors, 17(3):565, 2017.

[6] Robert Harle. A survey of indoor inertial positioning systems for pedestrians. IEEE Communications Surveys and Tutorials, 15(3):1281-1293, 2013.

[7] David Luenberger. An introduction to observers. IEEE Transactions on automatic control, 16(6):596-602, 1971.

[8] Bassem R Mahafza. Radar signal analysis and processing using MATLAB. Chapman and Hall/CRC, 2016.

[9] Roberto Manduchi and Sri Kurniawan. Mobility-related accidents experienced by people with visual impairment. AER Journal: Research and Practice in Visual Impairment and Blindness, 4(2):44-54, 2011.

[10] Abdelmoumen Norrdine, Zakaria Kasmi, and Jörg Blankenbach. Step detection for zupt-aided inertial pedestrian navigation system using foot-mounted permanent magnet. IEEE Sens. J, 16(1), 2016.

[11] World Health Organization. Blindness and vision impairment, accessed in november 2018. www.who.int/en/news-room/factsheets/detail/blindness-and-visual-impairment.

[12] Stefano Pisa, Emanuele Piuzzi, Erika Pittella, and Giuseppe Affronti. A fmcw radar as electronic travel aid for visually impaired subjects. In XXI Imeko World Congress, page 158, 2015.

[13] INSPEX H2020 project. Integrated smart spatial exploration system, accessed in november 2018. www.inspex.com.

[14] Tiana Rakotovao Andriamahefa. Integer Occupancy Grids: a probabilistic multi-sensor fusion framework for embedded perception. $\mathrm{PhD}$ thesis, Grenoble Alpes, 2017. 